\title{
(2) OPEN ACCESS \\ Role of club doctors in the mental health management of Australian rules football players: a Delphi study
}

\author{
Ben Weber, ${ }^{1}$ Jason Bos, ${ }^{2,3}$ Elizabeth Mary Clancy ${ }^{3},{ }^{3}$ Ranjit Menon, ${ }^{4,5}$ Tom Cross, ${ }^{1}$ \\ Kate Hall (i) 3,5
}

${ }^{1}$ The Stadium Sports Medicine Clinic, Sydney, New South Wales, Australia

${ }^{2}$ Mental Health Management, Sydney, New South Wales, Australia

${ }^{3}$ School of Psychology, Deakin University Faculty of Health, Burwood, Victoria, Australia ${ }^{4}$ Department of Psychiatry, University of Melbourne, Melbourne, Victoria, Australia ${ }^{5}$ Australian Football League, Melbourne, Victoria, Australia

\section{Correspondence to} Dr Kate Hall, School of Psychology, Deakin University Faculty of Health, Burwood, VIC 3125, Australia

kate.hall@deakin.edu.au

Accepted 2 October 2021
Check for updates

(C) Author(s) (or their employer(s)) 2021. Re-use permitted under CC BY-NC. No commercial re-use. See rights and permissions. Published by BMJ.

\section{To cite: Weber $B$,}

Bos J, Clancy EM, et al.

Br J Sports Med Epub ahead

of print: [please include Day

Month Year]. doi:10.1136/

bjsports-2021-104388

\section{ABSTRACT}

Professional team athletes experience a range of mental health problems, both sports and non-sports related. However, there is limited information available for those charged with responsibility for managing these mental health conditions, particularly within the context of professional sporting clubs. This paper reports on consensus findings from a study of club doctors, who are primary care providers for professional team athletes within a specific code, the Australian Football League (AFL). Drawing on findings from a systematic literature search, a two-round Delphi procedure was used to develop a consensus on best practice for managing mental health conditions for club doctors as primary care providers for professional team athletes. Participants in this study were current and former club doctors employed in professional AFL clubs across Australia, with 28 doctors participating across two survey rounds. Overall, 77 statements were presented, with 50 endorsed as essential or important by $\geq 80 \%$ of the participants across the two rounds. Primary themes across nine domains include: (1) Prevention and Mental Health Promotion Activities; (2) Screening; (3) Engaging External Specialists; (4) Duty of Care; (5) Treatment: Assessment, Treatment and Case Coordination; (6) Communication; (7) Confidentiality; (8) Sleep Management and (9) Substance Use Management. This study is the first to offer club doctors working in professional team settings consensus guidelines for the management of mental health conditions, and the opportunity for greater clarification and consistency in role delivery.

\section{INTRODUCTION}

Elite athletes are not immune to developing mental health problems, with research indicating that they experience comparable rates of mental health disorders as the general population. ${ }^{12}$ Like the general community, athletes experience non-sport-related risks for the development of mental health disorders, such as adverse life events, age, gender and inadequate social support. ${ }^{3}$ However, participation in elite sport confers a unique combination of both sport and non-sport-related risk factors associated with mental health disorders, such as injuries including concussion, ${ }^{4}$ performance failure, ${ }^{5}$ overtraining, ${ }^{6}$ social pressures to perform and stigmatisation and denial of mental health disorders. $^{7}$ Moreover, years of active participation in elite competition commonly overlap with peak age of onset for most mental health disorders. ${ }^{8}$ Together, these factors suggest that tailored and context-specific approaches are required to effectively respond to and manage the mental health of elite athletes.

Despite increasing research into elite athlete mental health and well-being, evidence regarding best practice management of mental health disorders remains limited. A recent review by the International Olympic Committee (IOC) ${ }^{9}$ highlighted a need for more standardised, evidence-based approaches to elite athlete mental health diagnosis and management. While research into professional team-based athletes' mental health has increased, ${ }^{10}$ the majority of existing empirical studies focus on collegiate teams and their athletes ${ }^{11}$ whose roles differ substantially from professional athletes with respect to their performance context, public profile and media scrutiny. As such, evidence-based guidelines into the management of mental health disorders in professional, team-based athletes are needed, to inform responses of team-based healthcare staff and to ultimately improve the management of mental health disorders in professional athletes. $^{12}$

The Australian Football League (AFL) is the preeminent professional league for Australian rules football, with 18 men's and 14 women's teams across mainland Australia. AFL club doctors are front-line providers of all aspects of player healthcare, including mental health. Although club doctors are not expert mental health clinicians, they have a duty of care for players' mental health and well-being. This is particularly important given the significant interplay between mental and physical health outcomes. ${ }^{9}$ Club doctors are well positioned to play a leadership role in the recognition, initial management and co-ordination of players' mental healthcare. However, there is an absence of consensus guidelines on how best to achieve this. This has resulted in inconsistent industry responses to important domains relevant to mental health identification and management, including use of screening and assessment tools, risk identification and management, treatment provision, case coordination, sleep management and addressing substance use. Moreover, management of player mental health within the broader context of a professional team elicits a host of additional considerations. These include managing confidentiality, navigating public and media scrutiny, involving multiple stakeholders in management planning including families, coaching and performance staff, 
and remaining aware of the implications for team performance and well-being.

There is thus a need to establish consensus about best practice and the role and responsibilities of club doctors in the mental health management of professional athletes in the AFL, which this study set out to achieve.

\section{METHOD}

\section{Methodology and survey development}

This study employed a modified Delphi methodology ${ }^{13}{ }^{14}$ to develop consensus on the role of club doctors in addressing and managing professional player mental health.

A systematic search of relevant databases (ie, Medline, PsycINFO, Embase and SportDiscus) was conducted in December 2019 using the following search terms: mental health ("mental health") OR ("addict*”) AND elite athletes ("elite athlete*") OR ("team sport") OR ("elite sport") AND treatment/management ("intervention"”) OR ("treatment"”) OR ("screen"”) OR ("management"). No date exclusions were applied, but only English-language papers were reviewed. This yielded a total of 279 studies, of which 257 were excluded based on title and/or abstract, resulting in a total of 22 papers which were reviewed for relevant content.

Using findings from the above literature review, alongside author expertise, an initial list of potential club doctor actions and roles were generated by the authors. Statements were reviewed to ensure clarity, limiting each statement to a single idea and providing alternatives where relevant. Statements were clustered thematically to reflect mental health promotion, screening and risk identification, treatment of mental health disorders, communication and information management. Additional items were added in a second-round survey, based on first-round participant suggestions. A full list of survey items is provided in table 1 .

\section{Participants}

Eligible participants for this study were registered club doctors currently or previously working with professional men's and women's AFL teams. To preserve anonymity, information regarding club affiliation, age and gender was not requested. A sample of 20 participants has been deemed sufficient in prior literature. ${ }^{15}$ This was exceeded, with 35 participants consenting and 28 participating in this study across two survey rounds. This represents $56 \%$ of the estimated 50 AFL club doctors currently affiliated with teams.

\section{Procedure}

After obtaining ethical approval from (blinded for review) University's Ethics in Human Research Committee, the initial survey was sent in April 2020 to participants via their professional group, the AFL Doctors Association (AFLDA). For all statements, participants were asked to rate the importance of the item on a 5 -point Likert scale. Response options were essential (1), important (2), depends (3), not important (4), should not be included (5), with an option to nominate 'do not know'. Participants could also add additional items via free-text response options, if they felt important areas were unaddressed.

After the first-round survey was completed, statements endorsed as 'essential' or 'important' by more than $80 \%$ of respondents were retained, while statements endorsed as 'essential' or 'important' by less than $60 \%$ of respondents were rejected. The remaining statements, in addition to newly proposed items, were presented to participants, again via the AFLDA, in a second-round survey in June 2020. The same criteria for retention and/or rejection were applied. As such, consensus represents the view of more than $80 \%$ of respondents across two survey rounds.

\section{RESULTS}

\section{Characteristics of participants}

A total of 28 participants completed the first-round survey; of these, $22(79 \%)$ completed the second-round survey. The majority of participants were currently working as AFL club doctors (96\%) and reported considerable breadth of experience, with 39\% having worked for more than ten years, $11 \%$ having worked for 5-10 years, 25\% between 2-5 years and 25\% for less than 2 years.

\section{Consensus}

The responses provided by club doctors fell within nine domains: (1) prevention and mental health promotion activities; (2) screening and risk identification; (3) engaging external specialists; (4) duty of care; (5) assessment, treatment and case coordination; (6) communication; (7) confidentiality; (8) sleep management and (9) substance use management. Table 1 details specific activities included under each domain and the level of agreement.

\section{DISCUSSION}

This study used a modified Delphi procedure to establish consensus about the role of club doctors in the mental health management of elite athletes in professional teams. Prevention and mental health promotion activities as well as regular mental health screening were identified as within the scope of the club doctor role. The participants strongly endorsed that club doctors should be aware of and engaged in whole-of-club mental health promotion activities, which should be evidence based, multidisciplinary and delivered by those with relevant expertise. In line with these views, a comprehensive mental health framework has been proposed ${ }^{3}$ for promotion of athlete mental health and wellbeing. This framework suggests that preventative components, such as mental health literacy training and screening tools are essential components within any elite or professional sporting context.

There was consensus that valid, reliable and standardised screening tools should be used at specific time frames in the athlete's career, namely, recruitment, preseason, end of season and end of contract or retirement, as well as critical life events or stressors. These views align with recommendations for standardised mental health screening tools by the IOC, who have developed the Sport Mental Health Assessment Tool and the Sport Mental Health Recognition Tool. This standardised assessment tool is one example of an athlete specific screening tool that could be implemented in sporting programmes for early identification of mental health symptoms. ${ }^{16}$ It was noted that additional mental health screening in the case of concussion was not sufficiently endorsed to meet acceptance criteria (78.3\%). Existing AFL concussion protocols mandate use of the SCAT ${ }^{17}$ which incorporates brief screening for psychological symptoms, and may be deemed sufficient. However, we also note emerging evidence of associations between concussion and mental health symptoms, ${ }^{18} 19$ and this result may suggest a need for further training for club doctors in this area.

The participants strongly agreed that club doctors ultimately held the duty of care for player mental health; however, it was also agreed that within each club, a nominated health 


\section{Prevention and mental health promotion activities}

1.1. Club doctors should be aware of all whole of club promotion and prevention programmes.

1.2. Club doctors should be engaged in whole of club mental health promotion and prevention programmes.

1.3. Whole of club mental health promotion and prevention programmes should be delivered by appropriately qualified professionals.

1.4. Club doctors should contribute their specific expertise as relevant to the design and delivery of promotion and prevention programmes.

1.5. All prevention and health promotion activities should be evidence based and multidisciplinary.

1.6. Providers of mental healthcare develop a rapport with athletes, even if this is outside professional consultations, given the importance placed by athletes on establishing relationships with providers to facilitate help seeking.

1.7. When working with elite athletes, club doctors should reconceptualise 'mental toughness' as 'mental fitness' to help to decrease perceptions of stigma around working with sport psychologists and talking about mental health.

\section{Screening and risk identification}

2.1. Each club has at least one nominated health professional with relevant expertise, who is responsible for mental health screening.

2.2. Screening should involve suitable valid and reliable standardised screening tools for mental health disorder.

2.3. Screening tools should include population tools, such as the PHQ), DASS21, K10 and/or AUDIT.

2.4. Screening tools should include athlete specific screening tools, such as the Athlete Stress Inventory or the Barron Depression Screener for Athletes.

2.5. In applying screening tools, for initial screening, compare results with population norms.

2.6. In applying screening tools for subsequent screening, compare results with individual's own baseline.

2.7. Screening should be conducted regularly.

2.8. Minimum screening is indicated at recruitment.

2.9. Minimum screening is indicated at preseason/end of season.

2.10. Minimum screening is indicated at mid-season.

2.11. Minimum screening is indicated at end of contract/retirement.

2.12. Additional screening is required as indicated in case of injuries.

2.13. Additional screening is required as indicated in case of concussion.

2.14. Additional screening is required as indicated in case of critical life events/stressors.

3. Engaging external specialists

3.1. If screening is indicated, a qualified mental health professional should conduct a comprehensive psychosocial assessment. This may involve one or more specialists as needed.

3.2. The club doctor must be aware of and engaged in coordination of external mental health assessment.

3.3. The club doctor is responsible for making referrals for external mental health assessment.

3.4. Club doctors should consider psychological, as well as physical factors, when treating and coordinating care for injured players.

3.5. If a player is referred to an orthopaedic or neurosurgeon, they should not be relied on to address the psychological issues related to injury, surgery or rehab of an athlete.

3.6. A qualified mental health professional should be engaged for assessment and management of any associated psychological issues.

\section{Duty of care}

4.1. The club doctor holds duty of care for the player's mental health.

4.2. A nominated club health professional (either the club doctor or another suitable mental health professional internal to the club) is the case coordinator.

4.3. If the club doctor who holds duty of care refers to a specialist psychiatrist, they share duty of care for the player's mental health.

\section{Assessment, treatment and case coordination}

5.1. If the club doctor has appropriate clinical expertise, they can be responsible for delivery of comprehensive assessment, treatment plan, specialised pharmacotherapy management and ongoing evidence-based psychological interventions.

5.2. If the club doctor does not have appropriate clinical expertise, the club doctor is responsible for coordination of comprehensive assessment and treatment plan, including specialised pharmacotherapy management and evidence-based psychological interventions. 5.3. Players should be discouraged from playing through distress, whether physical or mental.

5.4. If the club doctor has appropriate clinical expertise, they can be responsible for primary delivery of evidence-based psychological interventions.

5.5. If the club doctor does not have appropriate clinical expertise, they are responsible for coordination of communication between multidisciplinary providers and documentation.

5.6. This coordination role can be delegated to the case coordinator, but the club doctor must maintain duty of care and oversight of treatment progress.

5.7. Secondary and tertiary management: the club doctor is responsible for referral to appropriate external expertise, including IP admissions and residential programmes.

5.8. If the club doctor has appropriate clinical expertise, they can be responsible for ongoing mental health delivery of evidence-based psychological interventions.

5.9. If the club doctor does not have appropriate clinical expertise, they are responsible for coordination of communication between multidisciplinary providers and documentation.

5.10. This role can be delegated to the case coordinator, but the club doctor must maintain duty of care and oversight of treatment progress.
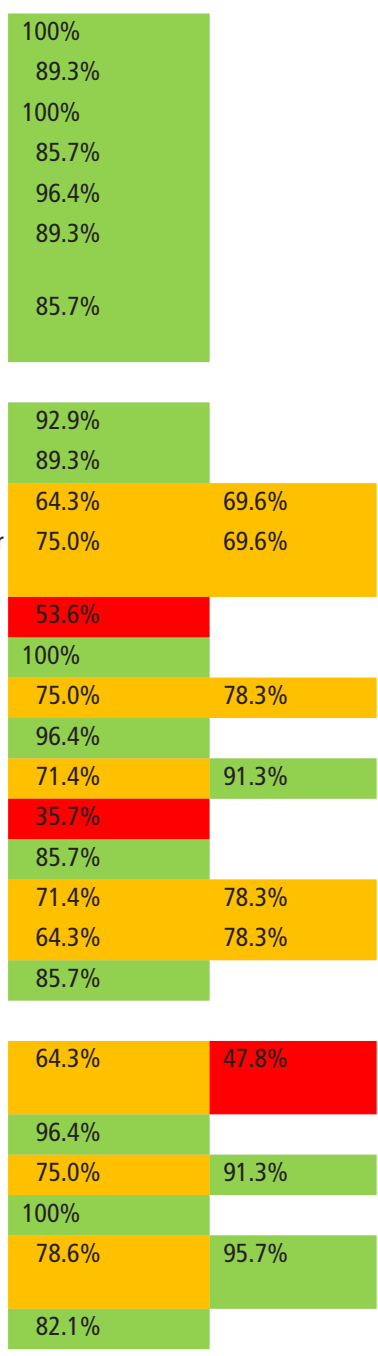

$89.3 \%$
$100 \%$
$96.4 \%$

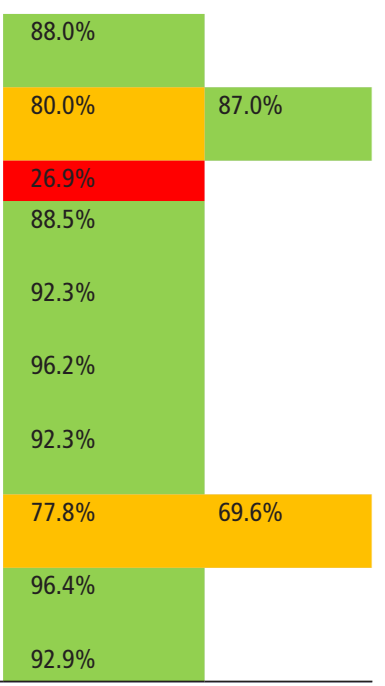

Continued 
5.11. The club doctor should engage secondary referrals and consultations with trusted expertise as needed to support delivery.

5.12. Case coordination meetings should be led by the nominated case coordinator.

5.13. The club doctor is responsible for providing a discharge summary, with player consent, on club transfer, retirement or delisting to a designated physician.

\section{Communication}

6.1. The club doctor should be aware of the club's social media policy.

6.2. The club doctor should be involved in a decision to notify, or not notify, the media of a player's mental health disorders.

6.3. The club doctor should not communicate directly with the media about players.

6.4. The club doctor should ensure adequate support is provided to a player when unwanted media attention is gained.

6.5. Club doctors should take advantage of social media and online platforms to disseminate knowledge about protecting the mental health of elite athletes.

6.6. The club doctor should encourage a player to communicate to the media/public about a mental health disorder.

6.7. The club doctor should encourage the involvement of family and friends in the management of a player's mental health disorder.

6.8. Key family members should be present when the club doctor delivers individual or group interventions regarding alcohol or substance use.

6.9. The club doctor or case coordinator should be the point of contact between the club and the family and friends of a player with a mental health disorder.

6.10. The club doctor or case coordinator should communicate updates on a player's mental health disorder to the coaching staff.

6.11. Education programmes should be made available to coaching staff to enhance their understanding of mental health disorders and needs and to assist them to facilitate help-seeking among players.

6.12. Clubs should encourage and actively facilitate a positive relationship between players and their mental health staff.

6.13. Clubs should ensure all staff who have contact with players are educated to increase their mental health literacy.

\section{Confidentiality}

7.1. Club doctors should be familiar with all relevant Commonwealth, State and Territory legislation, particularly where relevant to private and medical records. If in doubt, club doctors should consult with the AMA or their medical indemnity insurer.

7.2. Other than exceptional circumstances permitted or required by law (see below), a club doctor should not, without the patient's express up-to-date written consent, release medical records to persons other than the patient unless the patient would reasonably expect such disclosure to take place, in accordance with relevant privacy legislation. For example, it is likely a patient who has consented to the collection of their personal information for their healthcare may reasonably expect the doctor to share the patient's medical record among the treating healthcare team.

7.3. Club doctors should base any decision to disclose a patient's medical records to a third party on considerations of individual well-being of the patient, other identifiable individuals or groups and the public interest.

7.4. Where possible, any decision to disclose information should be discussed with the patient, and the nature of the discussion, the agreed level of disclosure and implications, should be documented in the medical record, including whether the patient consented to this disclosure. 7.5. Where disclosure of medical records is required by a third party under legislation, as in warrants, subpoenas or court orders, the club doctor should make all possible efforts to notify the individual of this occurrence and seek consent if possible.

\section{Sleep management}

8.1. Treating comorbid mental health disorders will likely be unhelpful unless any primary sleep disorder is treated

8.2. The club doctor or appropriate club personnel should have the ability to track how much sleep a player is getting.

8.3. The club doctor should provide sleep hygiene advice to players.

8.4. The club doctor should encourage sleep as part of a healthy training protocol.

8.5. Non-pharmacological treatment (such as CBT) for insomnia is preferred over medication.

8.6. Melatonin should be the first line pharmacological agent for players with insomnia.

8.7. The club doctor should only prescribe sleep aid medication according to evidence-based guidelines.

\section{Substance use and addiction management}

9.1. The club doctor's role is to provide evidence-based treatment for alcohol use disorders.

9.2. The club doctor's role is to coordinate evidence-based treatment for alcohol use disorders.

9.3. The club doctor's role is to provide evidence-based treatment for gambling disorder.

9.4. The club doctor's role is to coordinate evidence-based treatment for gambling disorder.

9.5. The club doctor's role is to provide evidence-based treatment for substance-use disorder.

9.6. The club doctor's role is to coordinate evidence-based treatment for substance-use disorder.

9.7. The club doctor should include clinical urine testing in the treatment and behavioural management of players with a substance use disorder.

9.8. The club doctor is the primarily responsible for the decision stand a player down who has an uncontrolled alcohol, gambling or substance use disorder.

9.9. The club doctor should coordinate a residential stay for a player who has an uncontrolled substance use disorder.

\begin{tabular}{|ll}
\hline Round 1 & Round 2 \\
\hline $100 \%$ & \\
$96.4 \%$ & \\
$78.6 \%$ & $78.3 \%$ \\
\hline
\end{tabular}
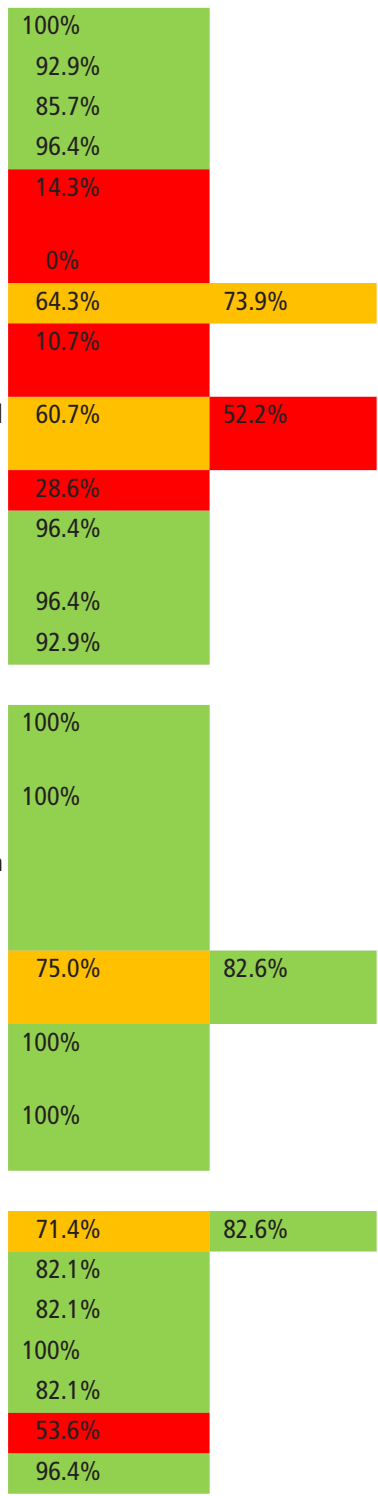

Green indicates $>80 \%$ endorsement of statement as essential or important.

Yellow indicates $60 \%-80 \%$ endorsement of statement as essential or important.

Red indicates $\leq 60 \%$ endorsement of statement as essential or important.

Initial wording of some survey items used the phrase 'mental health condition/s' but for consistency, this has been altered to 'mental health disorder/s' throughout.

AUDIT, alcohol use disorders identification test; DASS21, depression, anxiety and stress scale $21 ; \mathrm{K} 10$, kessler psychological distress scale; PHQ, patient health questionnaire. 
professional could be embedded to manage treatment coordination and ease administrative burdens on club doctors. This nominated treatment coordinator would liaise closely with the club doctor, ensuring overall integrated management, while bringing relevant additional expertise. This view is consistent with recommendations ${ }^{12}$ that appointed mental health officers could be a positive addition to multidisciplinary athlete support.

Regarding treatment, the participants agreed that if club doctors had relevant clinical expertise, they could conduct mental health assessments, manage pharmacotherapy and deliver initial evidence-based treatment. Club doctors who did not have relevant experience were still acknowledged to hold a duty of care and responsibility for oversight of treatment progress. As such, club doctors would hold responsibility for treatment coordination, which could be delegated to a nominated club professional, while the club doctor maintained oversight. Such an approach could facilitate the best use of professional expertise, while embedding consistent processes within the club and ideally contributing to an athlete-centred culture of care. ${ }^{20}$ When engaging external specialists, the participants agreed that club doctors should be responsible for referrals to external mental health professionals, while again maintaining oversight. They also endorsed a recommendation that concurrent referrals to mental health professionals should be considered when the principal injury is physical, given significant overlap between injury and mental health decline. ${ }^{4}$ They noted that physical injury specialists, such as orthopaedic surgeons or neurosurgeons, should not be assumed responsible for managing psychological sequalae of physical injuries.

The participants agreed that it was not the role of the club doctor to communicate with media, family or friends about a player's mental health disorder, nor did they believe that club doctors should be responsible for providing regular updates to coaching staff about a player's mental health. Such roles may create a conflict of interest for club doctors, ${ }^{21}$ and could potentially jeopardise athlete-centred focus of care. ${ }^{20}$ However, the participants agreed that club doctors should be involved in decisions to notify the media about a player's mental health disorder and be aware of the club's social media policy, so that actions can be taken in the best interests of the player's well-being. The participants endorsed standard protocols around patient confidentiality, following existing local legal and ethical protocols.

There was also agreement for a focus on building mental health literacy for all club staff, particularly those in coaching roles or other direct player contact, to understand mental health disorders and encourage help seeking, as supported by other experts. ${ }^{312}$ As expected, the participants agreed that club doctors must be familiar with and follow legal and professional protocols around privacy of medical information, basing all decisions to disclose information on consideration of well-being of the player, seeking consent for disclosure, and complying with legislative requirements.

Lastly, with respect to illicit drug policies, the participants agreed that the only role of the club doctor was to coordinate evidence-based treatments for alcohol use disorders, whereas directly providing treatment for alcohol, gambling and substance use was beyond the scope of their role. This last point highlighted the potential need for greater training in the management of gambling and substance use disorders for club doctors.

\section{Implications}

To date, support for elite athlete mental health has focused on building mental health literacy and awareness ${ }^{3}$; however, such an approach is insufficient without a comprehensive mental health system that can respond effectively to athlete needs, at the right time, the right place and with the right support. Findings from this study provide a starting point for club doctors to address the varied mental health needs of professional team athletes which has been lacking in published literature, and supports existing calls to action. ${ }^{3}$ This also supports investment in the critical role of club doctors to ensure quality provision of mental health programme delivery across prevention, early identification and intervention. $^{3}$

Investment in primary prevention and early intervention are an important part of stepped care approaches to mental health, and are an important part of a whole-of-club approach to ensure the club is capable of preventing athlete mental health disorders. We note that while club doctors hold a duty of care, they cannot be solely responsible for player mental health and well-being. Within a stepped care approach, ${ }^{22} 23$ engagement of expert tertiary resources is a valuable and efficient use of relevant expertise.

Within a given team or club, coaches, allied health staff, teammates and support staff, and external agencies such as the sport governing body, also have critical roles in management of player mental health within an integrated team. To achieve this, a focus on professional clubs as mentally healthy workplaces ${ }^{24}$ where risk factors are identified and addressed, and protective factors maximised, requires input from all organisational levels. Such an approach includes a culture of mental health literacy and awareness, stigma reduction and increased capacity for individuals other than club doctors, such as teammates, coaches and other support staff, to intervene and promote early help seeking. ${ }^{25} 26$ Nonetheless, it is noted that club doctors still hold a duty of care for the player's mental health, regardless of who performs dayto-day coordination roles. Therefore, specific and continuing mental health training should be a priority for club doctors in professional sporting contexts. While all medical practitioners receive mental health and psychiatry training during their medical degree, postgraduate training in mental health is limited. For sports and exercise registrars, the Australasian College of Sport and Exercise Physicians has recently developed a specific module, Sports Psychology for Sports Medicine, ${ }^{27}$ in which non-trainees can also enrol. However, regular, scheduled and ongoing professional mental health training for existing sports and exercise physicians may be helpful.

A second noteworthy implication concerns the need for access to both internal and external mental health expertise. Embedding mental health clinicians within a professional team can reduce stigma associated with needing to seek 'outside help'. ${ }^{28}$ They may also be best placed to hold responsibility for screening; a role which club doctors believed was needed, yet felt uncertain about who would hold primary responsibility. Crucially, should an athlete require intervention due to emergent concerns, including deteriorating pre-existing mental health disorders, it is imperative that the club has a critical incident response plan for how to best assess the situation, bring together relevant and suitably qualified professionals (especially in regard to lower prevalence conditions), formulate the planned strategy and implement a response, as per stepped care models. ${ }^{22}$ Additionally, specialist services and treatment for specific conditions, particular alcohol, substance use and gambling disorders, may require skills and expertise which are unlikely to be held by club doctors, and external providers would be necessary in such situations.

Regardless of specific screening tools used, there was consensus on the importance of screening, as part of a comprehensive early identification and intervention model of care ${ }^{29}$ again consistent 
with recommended best practice in mental health management for Olympic and other elite athletes. This offers the opportunity to triage athletes into levels of mental health risk, and then ensure access to services and resources based on their current profile, enabling the earliest and most effective mental health management.

Lastly, issues of confidentiality within the professional club setting remain challenging for club doctors. To support this, education for coaching staff and developing a psychologically safe workplace within the club, are likely to create a climate in which athletes feel that such information can be safely shared. ${ }^{30}$

\section{Limitations}

While our findings are of interest, we note some study limitations. First, although the sample size was within recommended guidelines for a Delphi procedure ${ }^{15}$ it may not reflect the experiences and perspectives of all club doctors. Second, information regarding the club doctors' education, training and experience in mental health was not obtained due to the ethical requirements for non-identifiable information to be collected. In addition, participants were limited to club doctors and did not include primary mental health clinicians. Although this privileges the opinion of club doctors, with varying levels of expertise in mental health, this research attempts to provide an initial consensus on the roles and responsibilities of the club doctor as determined by their own peer group. Further research is warranted to broaden the scope of opinion on the role of the club doctor through a multidisciplinary lens. Third, participants surveyed work within a professional team sport operating in a single nation, in wellresourced clubs, where there are strong regulatory frameworks around mental health treatment provision. As such, implications for other sports, or non-professional teams in less well-resourced environments, should be evaluated for applicability, as other professional team sports will have cultural-specific context and nuances. Lastly, this study is based on consensus, which means that if new information becomes available, suggested domains may need to be amended accordingly. Ideally, future research will enable these questions to be further explored.

\section{CONCLUSIONS}

Our findings identify the important role that club doctors play in the management of athlete mental health in professional Australian Rules football teams. Our consensus recommendations outline the ways in which club doctors can effectively address the mental health needs of their athletes, while acknowledging their limitations. A multidisciplinary team and a whole-of-club approach are needed to optimise prevention, early identification and treatment strategies to manage player mental health.

\section{Twitter Elizabeth Mary Clancy @lizclancy 123}

Acknowledgements The authors gratefully acknowledge the contributions and time of those club doctors who responded to and completed our surveys.

Contributors Conceptualisation: BW, KH and EMC; methodology: BW, KH and EMC; formal analysis: JB and EMC; investigation: BW, EMC, KH and TC, RM: data curation: JB and EMC; writing —original draft preparation: $\mathrm{EMC}, \mathrm{BW}$, JB; writingreview and editing, BW, EMC, JB, KH, TC and RM; supervision, $\mathrm{KH}$ and RM; project administration, EMC; funding acquisition: BW and EMC. All authors have read and agreed to the submitted version of the manuscript.

Funding This study was supported with funding from the AFL's Research Board and participant engagement was facilitated through the AFLDA.

Competing interests $\mathrm{KH}$ and RM were employed by the AFL as the Head of Mental Health and Wellbeing (KH) and AFL Chief Psychiatrist (RM) during this study, and have no other conflicts of interest to report. BW and TC are present and past medical doctors (respectively) at football clubs within the AFL competition, and have no other conflicts of interest to report. EMC and JB have no conflicts of interest to declare relevant to this paper. As such, only JB and EMC had access to the raw data and analyses to minimise any conflicts due to the roles of AFL staff and club staff.

\section{Patient consent for publication Not applicable.}

Ethics approval The study was conducted according to the guidelines of the Declaration of Helsinki, and approved by the Human Research Ethics Committee of Deakin University, Reference HEAG-H 155-2019, Approval dated November 2019.

Provenance and peer review Not commissioned; externally peer reviewed.

Open access This is an open access article distributed in accordance with the Creative Commons Attribution Non Commercial (CC BY-NC 4.0) license, which permits others to distribute, remix, adapt, build upon this work non-commercially, and license their derivative works on different terms, provided the original work is properly cited, appropriate credit is given, any changes made indicated, and the use is non-commercial. See: http://creativecommons.org/licenses/by-nc/4.0/.

\section{ORCID iDs}

Elizabeth Mary Clancy http://orcid.org/0000-0002-0167-7203

Kate Hall http://orcid.org/0000-0001-8648-0313

\section{REFERENCES}

1 Gouttebarge V, Castaldelli-Maia JM, Gorczynski P, et al. Occurrence of mental health symptoms and disorders in current and former elite athletes: a systematic review and meta-analysis. Br J Sports Med 2019;53:700-6.

2 Rice SM, Purcell R, De Silva $S$, et al. The mental health of elite athletes: a narrative systematic review. Sports Med 2016;46:1333-53.

3 Purcell R, Gwyther K, Rice SM. Mental health in elite athletes: increased awareness requires an early intervention framework to respond to athlete needs. Sports Med Open 2019:5:46.

4 Rice SM, Parker AG, Rosenbaum S, et al. Sport-related concussion and mental health outcomes in elite athletes: a systematic review. Sports Medicine 2018:48:447-65.

5 Hammond T, Gialloreto C, Kubas H, et al. The prevalence of failure-based depression among elite athletes. Clin J Sport Med 2013;23:273-7.

6 Frank R, Nixdorf I, Beckmann J, et al. Depression among elite athletes: prevalence and psychological factors. Deut Z Sportmed 2013;64:320-6.

7 Hughes L, Leavey G. Setting the bar: athletes and vulnerability to mental illness. $\mathrm{Br}$ J Psychiatry 2012;200:95-6.

8 Kessler RC, Berglund P, Demler O, et al. Lifetime prevalence and age-of-onset distributions of DSM-IV disorders in the National comorbidity survey replication. Arch Gen Psychiatry 2005;62:593-602.

9 Reardon CL, Hainline B, Aron CM, et al. Mental health in elite athletes: international Olympic Committee consensus statement (2019). Br J Sports Med 2019;53:667-99.

10 Psychological issues related to illness and injury in athletes and the team physician: a consensus Statement-2016 update. Med Sci Sports Exerc 2017;49:1043-54.

11 Schinke RJ, Stambulova NB, Si G, et al. International society of sport psychology position stand: Athletes' mental health, performance, and development. Int J Sport Exerc Psychol 2018;16:622-39.

12 Henriksen K, Schinke R, Moesch K, et al. Consensus statement on improving the mental health of high performance athletes. International Journal of Sport and Exercise Psychology 2020;18:553-60.

13 Adler M, Ziglio E. Gazing into the oracle: the Delphi method and its application to social policy and public health. Jessica Kingsley Publishers, 1996.

14 Jorm AF. Using the Delphi expert consensus method in mental health research. Aust $N$ Z J Psychiatry 2015;49:887-97.

15 Akins RB, Tolson H, Cole BR. Stability of response characteristics of a Delphi panel: application of bootstrap data expansion. BMC Med Res Methodol 2005;5:37.

16 Gouttebarge V, Bindra A, Blauwet C, et al. International Olympic Committee (IOC) sport mental health assessment tool 1 (SMHAT-1) and sport mental health recognition tool 1 (SMHRT-1): towards better support of athletes' mental health. Br J Sports Med 2021:55:30-7.

17 Sport concussion assessment tool - 5th edition. Br J Sports Med 2017;51:851-8.

18 Rice SM, Parker AG, Rosenbaum S, et al. Sport-Related concussion and mental health outcomes in elite athletes: a systematic review. Sports Med 2018;48:447-65.

19 Gouttebarge V, Kerkhoffs GMMJ. Sports career-related concussion and mental health symptoms in former elite athletes. Neurochirurgie 2021;67:280-2.

20 Dijkstra HP, Pollock N, Chakraverty R, et al. Managing the health of the elite athlete: a new integrated performance health management and coaching model. Br J Sports Med 2014;48:523-31.

21 Peer KS. The Changing Context of Sport and Medicine's Social Contract with Society: Implications for Sports Medicine Ethics. Int J Athletic Therap Train 2017;22:5-10.

22 Bower P, Gilbody S. Stepped care in psychological therapies: access, effectiveness and efficiency. narrative literature review. Br J Psychiatry 2005;186:11-17.

23 Cross SP, Hickie I. Transdiagnostic stepped care in mental health. Public Health Res Pract 2017;27. doi:10.17061/phrp2721712. [Epub ahead of print: 27 Apr 2017].

24 Harvey S, Joyce S, Tan L. Developing a mentally healthy workplace: a review of the literature. Sydney: National Mental Health Commission, 2014: 73. 
25 Gulliver A, Griffiths KM, Christensen H. Barriers and facilitators to mental health helpseeking for young elite athletes: a qualitative study. BMC Psychiatry 2012;12:157.

26 Wahto RS, Swift JK, Whipple JL. The Role of Stigma and Referral Source in Predicting College Student-Athletes' Attitudes Toward Psychological Help-Seeking. J Clin Sport Psychol 2016;10:85-98.

27 ACSEP. Sports Psychology for Sports Medicine [Training Module]. Australia: Australasian College of Sport and Exercise Physicians, 2021. www.acsep.org.au/page/ resources/acsep-education/acsep-academic-modules
28 Morse ED. Sports Psychiatrists Working in College Athletic Departments. In: Baron DA, Reardon CL, Baron SH, eds. Clinical sports psychiatry, 2013: 201-14.

29 Moesch K, Kenttä G, Kleinert J, et al. FEPSAC position statement: mental health disorders in elite athletes and models of service provision. Psycho/ Sport Exerc 2018;38:61-71.

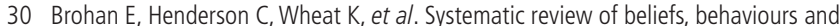
influencing factors associated with disclosure of a mental health problem in the workplace. BMC Psychiatry 2012;12:11. 